\title{
Determination of Deformable Image Registration Algorithms for Accumulating Dose in Carbon-ion Radiotherapy for Pancreatic Cancer
}

\author{
YANG LI $^{1,2}$, YOSHIKI KUBOTA ${ }^{3}$, MASAHIKO OKAMOTO ${ }^{3}$, SHINTARO SHIBA $^{3}$, \\ SHOHEI OKAZAKI ${ }^{3}$, TOSHIAKI MATSUI ${ }^{1}$, SHUNICHIRO KOMATSU ${ }^{3}$ and TATSUYA OHNO ${ }^{3}$ \\ ${ }^{1}$ Graduate School of Medicine, Gunma University, Maebashi, Japan; \\ ${ }^{2}$ Department of Radiation Oncology, Harbin Medical University Cancer Hospital, Harbin, P.R. China; \\ ${ }^{3}$ Gunma University Heavy Ion Medical Center, Maebashi, Japan
}

\begin{abstract}
Background/Aim: This study aimed to determine appropriate deformable image registration (DIR) algorithms for pancreatic cancer patients undergoing carbon ion radiotherapy (CIRT). Patients and Methods: The performance of three types of DIR algorithms, including intensity-based DIR (iDIR), contour-based DIR (cDIR), and hybrid DIR ( $h D I R)$ were evaluated using seventy-one $C T$ images from eight pancreatic cancer patients. Both the geometry of the CTV and subsequent dose warping discrepancies were evaluated using the dice similarity coefficient (DSC) and the difference in V95. Results: cDIR and hDIR had superior performance than iDIR in both DSC and V95 $(p<0.0001)$. iDIR caused accumulated dose to be underestimated by $5 \%$ in the median CTV V95 compared to the other methods. Conclusion: hDIR and cDIR can be utilized to assess the accumulated dose in CIRT for pancreatic cancer. iDIR can be considered when the obtained DSC is greater than 0.89.
\end{abstract}

Carbon ion radiotherapy (CIRT) is an effective technology for use in patients with pancreatic cancer due to its unique physical and biological characteristics. The Bragg peak and sharp lateral dose penumbra allows for an excellent dose distribution in the target, while sparing healthy tissues (1). However, this property also makes it highly sensitive to the

This article is freely accessible online.

Correspondence to: Yoshiki Kubota, Ph.D., Gunma University Heavy Ion Medical Center, 3-39-22 Showa-machi, Maebashi, Gunma 371-8511, Japan. Tel: +81 272208378, Fax: +81 272208379, e-mail: y_kubota@gunma-u.ac.jp

Key Words: Deformable image registration (DIR), accuracy evaluation, intensity-based DIR, contour-based DIR, hybrid DIR, pancreatic cancer, carbon ion radiotherapy, dose accumulation. position and anatomical uncertainties of the tumor and normal tissues over the course of treatment. A threedimensional imaging verification system is currently available in some carbon ion facilities (2); this system makes it possible to conduct dosimetric assessment based on daily CT images, and provides a possibility to perform adaptive radiotherapy with carbon ion beams. To trigger re-planning during adaptive RT, estimation of the cumulated dose is necessary to obtain the actual delivered dose over fractions. Therefore, it is important to select a tool with a sufficiently high accuracy to accumulate the dose.

A satisfactory image registration between moving and reference CT images is essential to evaluate the accumulated dose. In recent years, many image registration software and tools have been developed and implemented in clinical practice, in particular, deformable image registration (DIR). In contrast to rigid image registration (RIR), which is effective in cases where no anatomical change is expected, DIR can manage local distortion between two image sets (i.e., organ deformation and tumor movement) (3). Therefore, RIR is not considered to be appropriate for pancreatic cancer, where a large organ deformation in the gastrointestinal tract is common (4). Furthermore, the accuracy of image registration in dose warping is much lower in RIR than in DIR methods (5). Intensity-based DIR (iDIR) has been widely utilized for contour propagation, dose warping, and accumulation for various cancers (6). iDIR uses a variety of image intensity metrics, such as gray scale, mutual information, and cross correlation, to match the images. Therefore, iDIR can achieve high accuracy for organs with ample distinctive image features or high internal contrast $(7,8)$. Indeed, iDIR has been used to examine the accumulated doses for lung and liver cancer in $\operatorname{CIRT}(9,10)$. However, for pancreatic cancer, it is often difficult to identify the boundary between the tumor and surrounding soft tissues on CT images due to the low contrast, which may 
Table I. Patient characteristics.

\begin{tabular}{|c|c|c|c|c|c|c|c|c|}
\hline \multirow{2}{*}{$\begin{array}{l}\text { Patient } \\
\text { No. }\end{array}$} & \multirow[t]{2}{*}{ Gender } & \multirow{2}{*}{$\begin{array}{l}\text { Age } \\
\text { (year) }\end{array}$} & \multirow{2}{*}{$\begin{array}{l}\text { Tumor } \\
\text { position }\end{array}$} & \multirow{2}{*}{$\begin{array}{l}\text { Daily } \\
\text { CT sets }\end{array}$} & \multicolumn{2}{|c|}{ Planning CT } & \multicolumn{2}{|c|}{ Daily CT } \\
\hline & & & & & CTV (ml) & GTV (ml) & CTV (ml) & GTV (ml) \\
\hline 1 & Female & 50 & Body & 9 & 94.0 & 18.0 & $98.6(90.9-104.9)$ & $26.0(24.0-29.5)$ \\
\hline 2 & Male & 76 & Head & 9 & 158.1 & 21.3 & $156.1(150.3-168.0)$ & $22.7(20.0-24.8)$ \\
\hline 3 & Female & 83 & Head & 9 & 170.3 & 32.0 & $172.9(163.6-182.1)$ & $33.5(30.3-37.6)$ \\
\hline 4 & Male & 81 & Body & 9 & 202.0 & 29.0 & $174.1(157.0-204.2)$ & $27.3(26.1-31.4)$ \\
\hline 5 & Female & 51 & Head & 9 & 126.8 & 24.9 & $112.0(101.3-128.5)$ & $24.7(19.9-29.3)$ \\
\hline 6 & Female & 61 & Body & 9 & 127.6 & 23.9 & $129.7(117.0-134.1)$ & $25.6(21.5-28.0)$ \\
\hline 7 & Female & 78 & Body & 8 & 131.3 & 18.5 & $127.5(122.1-138.5)$ & $21.1(18.6-23.9)$ \\
\hline 8 & Male & 74 & Head & 9 & 165.8 & 37.2 & $169.3(158.7-179.3)$ & $42.8(37.2-48.2)$ \\
\hline
\end{tabular}

Data are presented as median (range). CT: Computed tomography; CTV: clinical target volume; GTV: gross tumor volume.

reduce the accuracy of iDIR. Additionally, the iDIR algorithm has limitations in the extent to which it can accurately match large heterogeneous deformations in regions without the aid of features between images (11-13). To reduce these uncertainties, contour-based DIR (cDIR) and hybrid DIR (hDIR) have been recently developed, which use only contours in images (cDIR), or both the contour and image intensity metrics (hDIR) to guide the registration. cDIR and hDIR appear to have the potential to improve the accuracy of DIR for pancreatic cancer. However, few studies have focused on testing the accuracy of DIR algorithms for pancreatic cancer, especially those based on CIRT $(5,14$, 15). Considering the steeper dose gradients of carbon ion beams, the uncertainties of DIR in anatomical structure registration may have greater effects on dose warping than conventional RT. Therefore, evaluation of the accuracy of DIR in CIRT is necessary. In our previous study, we demonstrated that cDIR and hDIR can obtain a better accuracy in dose warping than iDIR for CT image registration between different patient positions (supine and prone) (5), while a similar performance was observed between the same patient position. However, the most appropriate DIR method for dose accumulation was unclear due to the limited number of CT images studied.

In the current study, we evaluated the performance of iDIR, cDIR, and hDIR methods in eight patients with pancreatic cancer. To this end, we examined adaptive CIRT based on daily CT sets, and proposed a cut-off value for clinical DIR selection for dose accumulation by quantifying the discrepancy between the DIR algorithms.

\section{Patients and Methods}

Patient material. A total of eight pancreatic cancer patients treated with CIRT with a passive irradiation method were studied; the data were generated from the same patient group used in our previous study (5). In contrast to our previous study, where few CT sets were used, in the current study, all daily CT scans acquired on the treatment day were analyzed. However, to avoid uncertainties from the different positions, only the cases with supine position were selected; the details are shown in Table I. This study was approved by the Institutional Review Board (1564) of our facility, and the study was registered at the University Hospital Medical Information Network Clinical Trials Registry (UMIN-CTR trial number: 000029495).

Image acquisition. The planning CT images were acquired in the simulation room in the 2 weeks before treatment. Daily CT data sets were acquired by a separate CT (CT-on-rails) system on the treatment day under the same settings and conditions as the planning $\mathrm{CT}$ in the treatment room (Aquilion LB, Self-Propelled, Canon Medical Systems, Otawara, Japan). Only the CT images with supine position (the first nine fractions) were selected in this study. Thus, a total of eight planning $\mathrm{CT}$ sets was obtained as fixed (reference) $\mathrm{CT}$, and 71 daily CT sets were obtained as moving CT (one CT set was missing in case 7 due to equipment fault). The median (range) interval time between treatment days was 1 day (range $=1-5$ days).

Treatment planning. The contours of the clinical target volume (CTV) and gross tumor volume (GTV) on the planning CT images were used as the reference. To reduce uncertainties from the contour delineation, one radiation oncologist manually delineated the target volumes on each of the daily CT sets, which was subsequently checked by another experienced radiation oncologist. The GTV was determined via contrast-enhanced CT before treatment. To protect the gastrointestinal (GI) organs, additional margins were defined as planning organ at risk volume (PRV)-GI (GI boundary $+2 \mathrm{~mm}-\mathrm{GTV}$ ) at our facility. The CTV was defined as the GTV plus a $5 \mathrm{~mm}$ margin, including locoregional lymph nodes and neural plexus regions, minus the PRVGI margin. The planning target volume (PTV) was determined by adding a $3 \mathrm{~mm}$ margin to the CTV, excluding the PRV-GI margin. The treatment plan of four beam fields with a passive scattering technique was created using the $\mathrm{XiO}-\mathrm{N}$ system, which employs a pencil-beam algorithm (Elekta, Stockholm, Sweden and Mitsubishi Electric, Tokyo, Japan), and only one beam delivered on each treatment day. Our facility uses Gy as the clinical dose unit, which was calculated based on the physical dose and the relative biological effectiveness (RBE) (16). The prescribed dose for each fraction was 4.6 Gy (RBE), and a total of 12 fractions were applied to each patient. 


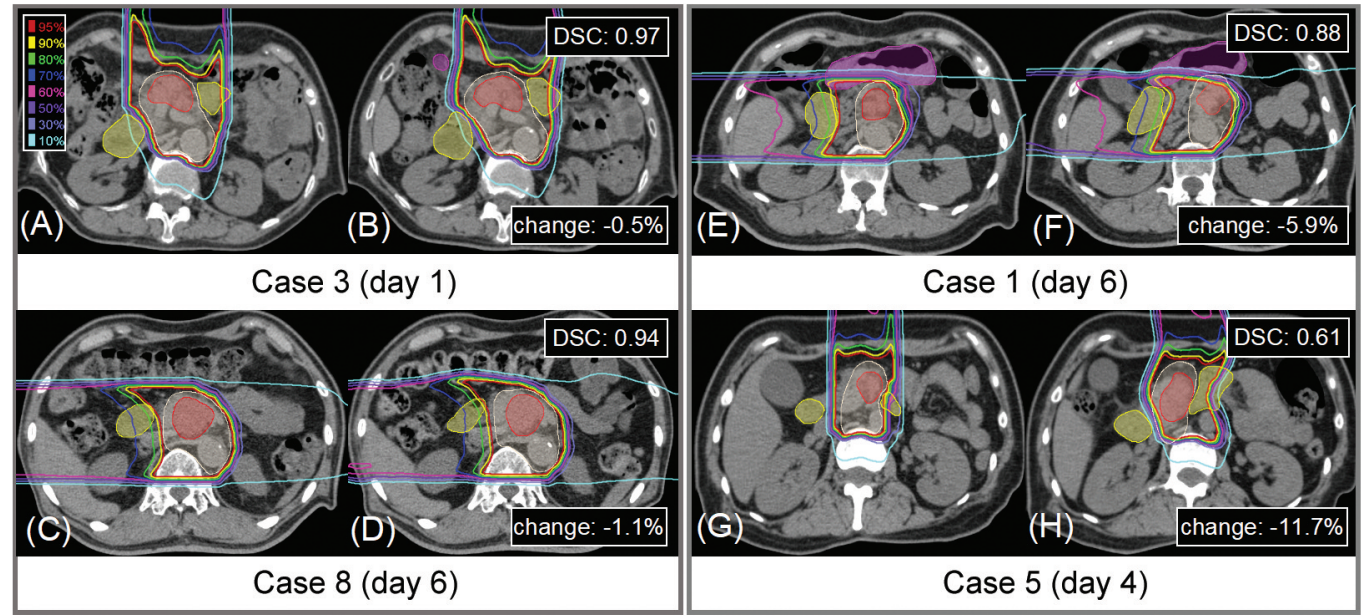

Figure 1. Example of dose distributions deformed via iDIR in four cases. The left panel in each column shows the dose distributions on one daily set of $C T$ images $(A, C, E, G)$, and the right panel shows the deformed doses via iDIR on planning $C T$ images $(B, D, F, H)$. The filled white, red, purple, and yellow show the clinical target volume (CTV), gross tumor volume (GTV), stomach, and duodenum, respectively. Change: Dose reduction in CTV receiving greater than $95 \%$ of the prescription dose (V95).

New dose distributions with the same angle as the irradiated beam in the first 9 fractions (angles: $0^{\circ}, 90^{\circ}$, and $270^{\circ}$ ) were generated independently with the same criteria as the treatment planning and used for the analysis. The last 3 fractions with prone position were excluded. The doses on daily CT images were used as the ground-truth doses.

Deformable image registration algorithm and evaluation parameters. The DIR algorithms were implemented using the VoxAlign Deformation Engine in MIM maestro (MIM Software Inc., Cleveland, OH, USA). Three types of DIR algorithms, including iDIR, cDIR, and hDIR, were applied to deform the daily $\mathrm{CT}$ images to the planning CT images. The iDIR algorithm is a freeform based deformable registration that assumes that voxel values (image intensity) of the same anatomical area are similar between CT images. The cDIR algorithm focuses solely on the contour information without depending on the image intensity, assuming that the target-volume changes are small. However, the hDIR algorithm, considers both the target structure and image intensity information (outside the contour). The deformation vector fields (DVFs) obtained after DIR between the planning CT and daily CT images were applied to the target contours and doses on the daily CT to validate the accuracy of the DIR. Delineated CTV and GTV were deformed separately for cDIR and hDIR, in order to avoid potential uncertainties caused by the deformation of multiple contours. In addition, before performing DIR, all daily CT images were rigidly registered in the corresponding planning $\mathrm{CT}$ image with manual tumor matching to improve the accuracy of DIR (17).

Structure correspondence was quantified with metrics of the dice similarity coefficient (DSC), which was calculated to evaluate the amount of volume overlap between each pair of contours using the following formula: DSC $=2(\mathrm{~V} 1 \cap \mathrm{V} 2) /(\mathrm{V} 1+\mathrm{V} 2)$, where $\mathrm{V} 1$ and $\mathrm{V} 2$ are the volumes of the contour on the planning $\mathrm{CT}$, and the contour deformed from the daily $\mathrm{CT}$ to the planning $\mathrm{CT}$, respectively. In this study, we only evaluated the CTV and GTV, while other contours, such as the stomach and duodenum, were not considered. Dose correspondence is another important index to validate the accuracy of DIR, and the dose distribution on each daily CT set was warped and transferred to the corresponding planning CT images using the DVF obtained from each registration. Then, dose differences in the daily and accumulated doses among different DIR algorithms were evaluated by dose-volume parameters such as CTV V95 and GTV V95 (the volume receiving greater than $95 \%$ of the prescription dose). In principle, the dose parameters cannot be preserved when the contour volumes are different between the CT images. However, they will be close when the volume changes are very small, and in this situation, a large parameter difference should indicate large errors. Because the CTV and GTV did not change significantly in this study, we considered that smaller dose parameter differences indicated a higher accuracy of DIR.

We used Wilcoxon signed rank test to evaluate the difference in the DSC and dose distributions between the DIR methods. A simple linear regression was used to examine the relationship between the DSC and the dose distributions for the three DIR methods, the dependence between the percentage of cases with a dose difference greater than $2 \%(5 \%)$ after iDIR, and the difference in the accumulated dose in respect to hDIR and cDIR. Receiver operating characteristic (ROC) curve analysis was performed for the DSC with a dose difference greater than $2 \%$, and $5 \%$ for iDIR. The cutoff value was determined when the sensitivity was $90 \% . p<0.05$ was considered statistically significant, and $p<0.0001$ was considered as highly statistically significant.

\section{Results}

Figure 1 shows an example of CTV dose distribution deformed via iDIR. The average \pm standard deviation of the DSC of the CTV for all cases with iDIR, hDIR, and cDIR were $0.88 \pm 0.06,0.94 \pm 0.01$, and $0.96 \pm 0.01$, respectively (Table II). hDIR and cDIR had a superior CTV overlap than iDIR 
Table II. Absolute dose difference in clinical target volume (CTV) receiving greater than 95\% of the prescription dose (V95) and dice similarity coefficient (DSC) of the CTV with different deformable image registration (DIR) algorithms.

\begin{tabular}{|c|c|c|c|c|c|c|c|c|c|c|c|}
\hline \multirow[t]{2}{*}{ No. } & \multicolumn{3}{|c|}{ iDIR (\%) } & \multicolumn{2}{|c|}{ hDIR (\%) } & cDIR (\%) & \multicolumn{3}{|c|}{ DSC } & \multicolumn{2}{|c|}{ Accumulated (\%) } \\
\hline & V95 & Ratio $^{\mathrm{a}}$ & Ratio $^{b}$ & V95 & Ratio $^{\mathrm{a}}$ & V95 & iDIR & hDIR & cDIR & hDIR-iDIR & cDIR-iDIR \\
\hline 1 & $1.4(0.6-5.9)$ & 44.4 & 11.1 & $0.6(0.1-1.8)$ & 0 & $0.2(0-0.7)$ & $0.89(0.88-0.93)$ & $0.94(0.93-0.95)$ & $0.95(0.94-0.96)$ & 3.1 & 3.5 \\
\hline 2 & $3.7(2.4-5.7)$ & 100 & 22.2 & $0.91(0.1-2.2)$ & 22.2 & $0.4(0.2-0.9)$ & 0.89 & $0.95(0.94-0.95)$ & $0.96(0.95-0.97)$ & 3.9 & 4.6 \\
\hline 3 & $2.1(0.5-5.9)$ & 55.6 & 11.1 & $0.3(0-1.3)$ & 0 & $0.3(0-0.7)$ & $0.89(0.86-0.97)$ & $0.94(0.93-0.98)$ & $0.96(0.95-0.98)$ & 4.1 & 4.1 \\
\hline 4 & $4.5(0.2-19.4)$ & 88.9 & 44.4 & $0.2(0.2-0.7)$ & 0 & $0.2(0.1-0.5)$ & $0.87(0.80-0.96)$ & $0.94(0.94-0.97)$ & $0.96(0.95-0.97)$ & 15.2 & 15.4 \\
\hline 5 & $7.5(0.3-18.4)$ & 77.8 & 66.7 & $0.3(0-1.4)$ & 0 & $0.3(0-0.7)$ & $0.81(0.61-0.87)$ & $0.93(0.92-0.94)$ & $0.95(0.95-0.96)$ & 12.8 & 13.2 \\
\hline 6 & $2.0(0.2-9.9)$ & 44.4 & 33.3 & $0.4(0-1.8)$ & 0 & $0.1(0-1.5)$ & $0.89(0.84-0.97)$ & $0.94(0.93-0.97)$ & $0.96(0.95-0.97)$ & 6.9 & 7 \\
\hline 7 & $3.1(1.9-8.6)$ & 75.0 & 25.0 & $0.5(0.3-1.1)$ & 0 & $0.1(0-0.3)$ & $0.86(0.84-0.94)$ & $0.94(0.93-0.97)$ & $0.96(0.95-0.96)$ & 4.8 & 5.2 \\
\hline 8 & $0.6(0-2.2)$ & 11.1 & 0 & $0.2(0.1-0.5)$ & 0 & $0.1(0-0.2)$ & $0.93(0.92-0.98)$ & $0.95(0.94-0.98)$ & $0.96(0.95-0.97)$ & 0.2 & 0.3 \\
\hline All & $\begin{array}{l}2.7 \\
(0-19.4) *\end{array}$ & 62.0 & 26.8 & $0.4(0-2.2)$ & 2.8 & $0.2(0-1.5)$ & $0.89(0.61-0.98)^{*}$ & $0.94(0.92-0.98)^{\mathfrak{f}}$ & $0.96(0.94-0.98)$ & $\begin{array}{l}4.5 \\
(0.2-15.2)\end{array}$ & $\begin{array}{l}4.9 \\
(0.3-15.4)\end{array}$ \\
\hline
\end{tabular}

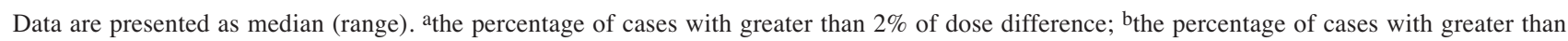
$5 \%$ of dose difference; $* p<0.0001$ compared with hDIR and cDIR; ${ }^{£} p<0.0001$ compared with cDIR.

Table III. Absolute dose difference in gross tumor volume (GTV) receiving greater than 95\% of the prescription dose (V95) and dice similarity coefficient (DSC) of the GTV with different deformable image registration (DIR) algorithms.

\begin{tabular}{|c|c|c|c|c|c|c|c|c|c|c|}
\hline \multirow[t]{2}{*}{ No. } & \multicolumn{3}{|c|}{ iDIR (\%) } & \multirow{2}{*}{$\frac{\text { hDIR (\%) }}{\text { V95 }}$} & \multirow{2}{*}{$\frac{\text { cDIR }(\%)}{\text { V95 }}$} & \multicolumn{3}{|c|}{ DSC } & \multicolumn{2}{|c|}{ Accumulated (\%) } \\
\hline & V95 & Ratio $^{a}$ & Ratio $^{\mathrm{b}}$ & & & iDIR & hDIR & cDIR & hDIR-iDIR & cDIR-iDIR \\
\hline 1 & $0(0-3.9)$ & 11.1 & 0 & $0.1(0-1.0)$ & $0.1(0-0.3)$ & $0.77(0.73-0.85)$ & $0.87(0.86-0.90)$ & $0.90(0.88-0.91)$ & 0.7 & 0.9 \\
\hline 2 & $0(0-0.3)$ & 0 & 0 & $0(0-0.3)$ & $0(0-0.1)$ & $0.81(0.76-0.87)$ & $0.88(0.85-0.90)$ & $0.91(0.90-0.92)$ & 0 & 0 \\
\hline 3 & $0.6(0-0.9)$ & 0 & 0 & $0.6(0.1-1.5)$ & $0.2(0-1.5)$ & $0.82(0.77-0.93)$ & $0.89(0.87-0.94)$ & $0.91(0.89-0.93)$ & 0.2 & 0.2 \\
\hline 4 & $0(0-0.6)$ & 0 & 0 & $0(0-0.5)$ & $0(0-0.5)$ & $0.86(0.78-0.93)$ & $0.88(0.85-0.94)$ & $0.90(0.89-0.94)$ & 0 & 0 \\
\hline 5 & $2.2(0.5-7.0)$ & 55.6 & 22.2 & $0.5(0.1-1.7)$ & $0.6(0-1.5)$ & $0.80(0.41-0.85)$ & $0.91(0.89-0.92)$ & $0.93(0.92-0.94)$ & 2.2 & 2.3 \\
\hline 6 & $0.2(0-3.1)$ & 11.1 & 0 & $0.2(0-1.1)$ & $0(0-0.8)$ & $0.87(0.83-0.94)$ & $0.92(0.91-0.95)$ & $0.93(0.93-0.95)$ & 0 & 0 \\
\hline 7 & $0(0-0.1)$ & 0 & 0 & $0(0-0.1)$ & $0(0-0)$ & $0.83(0.82-0.95)$ & $0.87(0.87-0.95)$ & $0.91(0.90-0.94)$ & 0 & 0 \\
\hline 8 & $0(0-0)$ & 0 & 0 & $0(0-0)$ & $0(0-0)$ & $0.87(0.82-0.94)$ & $0.92(0.90-0.94)$ & $0.94(0.92-0.95)$ & 0 & 0 \\
\hline All & $0(0-7.0)^{\dagger}$ & 9.9 & 2.8 & $0(0-1.7)$ & $0(0-1.5)$ & $0.83(0.41-0.95)^{*}$ & $0.90(0.85-0.95)^{£}$ & $0.91(0.88-0.95)$ & $0(0-2.2)$ & $0(0-2.3)$ \\
\hline
\end{tabular}

Data are presented as median (range). athe percentage of cases with greater than $2 \%$ of dose difference; bthe percentage of cases with greater than $5 \%$ of dose difference; $* p<0.0001$ compared with hDIR and cDIR; ${ }^{£} p<0.0001$ compared with cDIR; ${ }^{\dagger} p<0.05$ compared with hDIR and cDIR.

$(p<0.0001)$; although hDIR presented satisfactory DSCs, better DSCs were obtained in cDIR $(p<0.0001)$. However, decreased DSCs were observed for the GTV compared to the CTV, with iDIR, hDIR, and cDIR values of $0.82 \pm 0.07$, $0.89 \pm 0.03$, and $0.92 \pm 0.02$, respectively (Table III). iDIR had the worst DSC performance among the DIR methods. The dose differences in the CTV and GTV for all cases are shown in Figure 2. For the CTV V95, the dose changes were much smaller in cases with hDIR and cDIR than in those with iDIR (Figure 2A) $(p<0.0001)$. Most cases had a dose change of less than $2 \%$ when using cDIR and hDIR, especially for cDIR, and there was only one case in which the dose change was greater than $1 \%$. However, using iDIR, approximately $62 \%$ of cases had a change greater than $2 \%$, while $27 \%$ of cases had a change greater than $5 \%$ (Table II). It is important to note that an underestimated dose may be obtained following the application of iDIR (Figure 2B). For the GTV, the dose differences were small, and similar in all cases between DIR methods (Figure 2D). However, there were more extreme values with larger dose difference in cases using iDIR (Figure 2C), the details of which are shown in Tables II and III.

A negative dependence between the DSC and dose difference was observed in the CTV with iDIR (Pearson correlation coefficient (R): -0.64) (Figure 3A). However, the other two DIR methods had no such dependence. For the GTV, there was no dependence between the DSC and the dose difference in any 
A

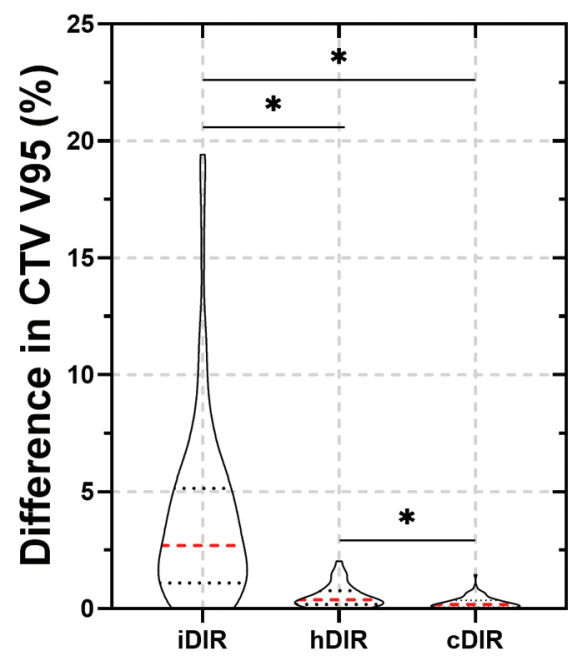

C

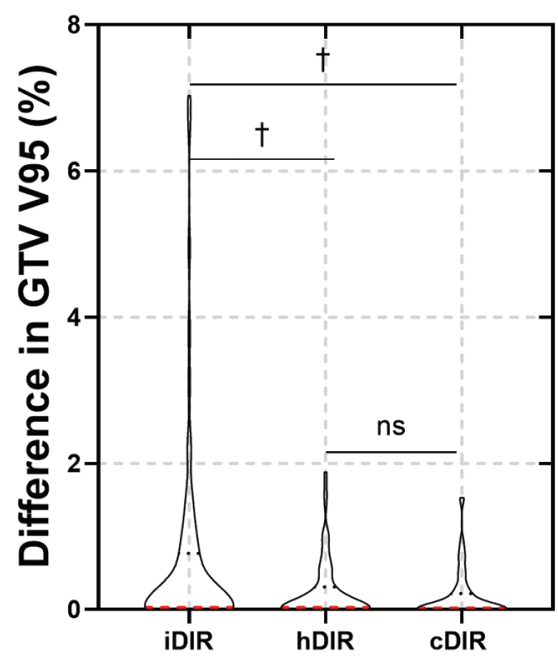

B

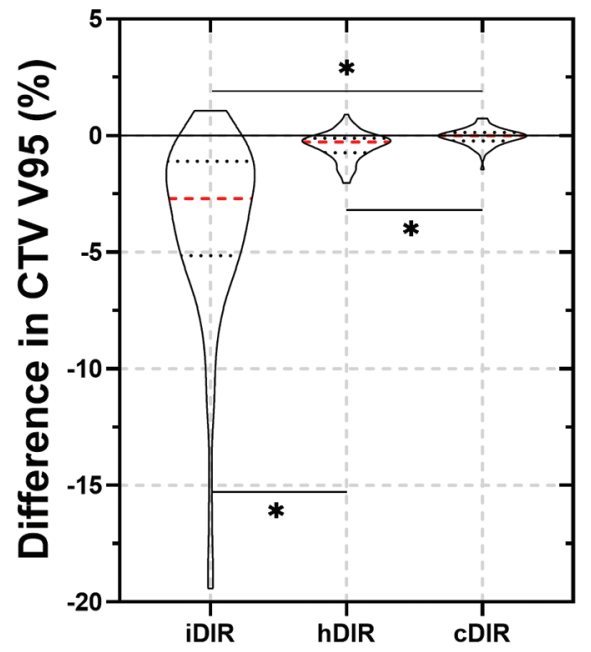

D

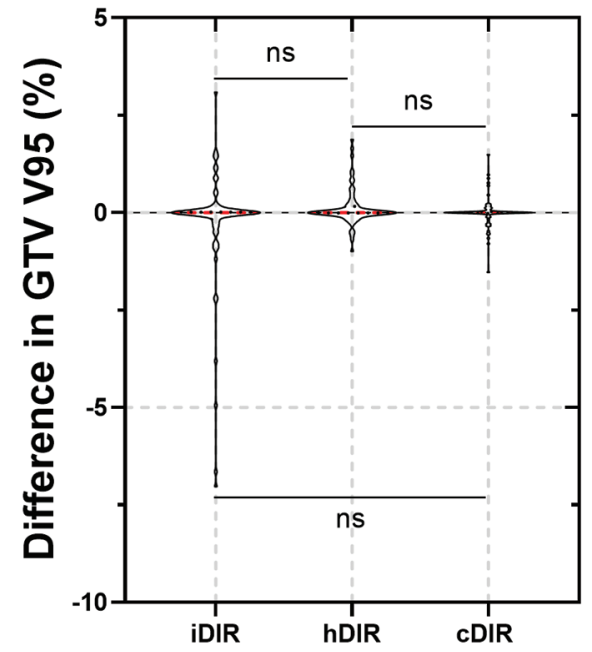

Figure 2. Violin plots for dosimetric difference in clinical target volume (CTV) and gross tumor volume (GTV) receiving greater than $95 \%$ of the prescription dose (V95). (A) and (C) show the absolute dose difference in CTV and GTV. The actual dose differences for CTV and GTV are shown in $(B)$ and $(D)$, respectively. The dotted red lines indicate the median dose changes, and the dotted black lines show the data at the $25^{\text {th }}$ and $75^{\text {th }}$ percentile. ${ }^{*} p<0.0001 ;{ }^{\dagger} p<0.05$.

of the DIR methods (Figure 3B). ROC was performed for DSC with greater than $2 \%$ and $5 \%$ dose differences in the CTV V95 after iDIR (Figure 4). The area under the curve was 0.82 and 0.84 , respectively, and the cut-off value was 0.91 and 0.89 (the sensitivity was $90 \%$ ), respectively; these results indicated that the dose changes would be greater than $2 \%$ if the DSC value is smaller than 0.91 , and the dose changes would be greater than $5 \%$ if the DSC is smaller than 0.89 .

Using iDIR, the median accumulated dose was reduced by approximately $5 \%$ in the CTV V95 compared to the other methods in this study (Table II). The dose difference was very small between hDIR and cDIR, and there was no significant difference in GTV between the DIR methods (Table III). Furthermore, a strong dependence was obtained between the percentage of cases with a dose difference greater than $5 \%$, and the accumulated dose difference in respect to the hDIR and cDIR $(\mathrm{R}=0.90)$ (Figure 5).

\section{Discussion}

Limited studies have evaluated the performance of DIR algorithms based on CIRT. To the best of our knowledge, this is the first study to determine a cut-off DSC value for iDIR for an accumulating dose in radiotherapy for pancreatic cancer. 
A

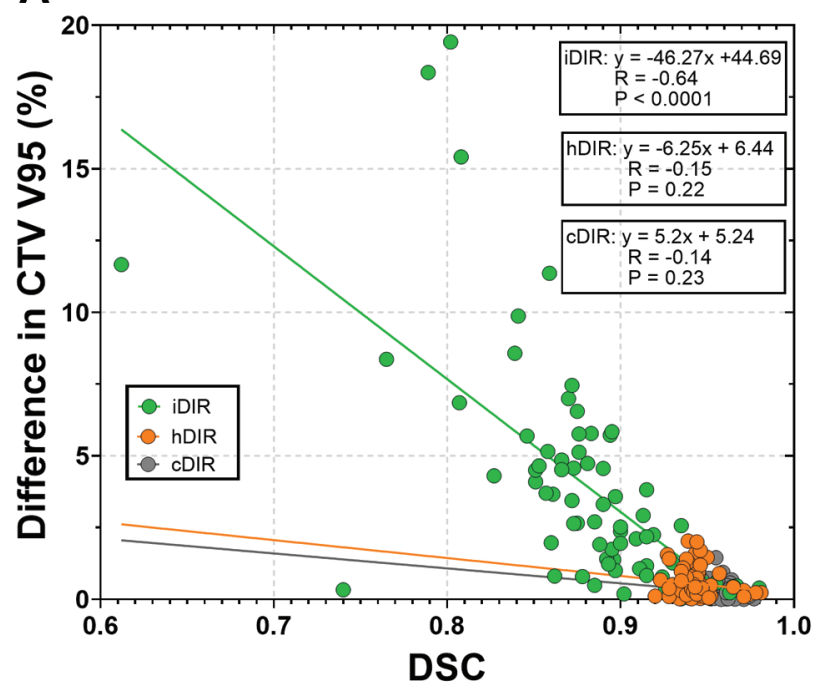

B

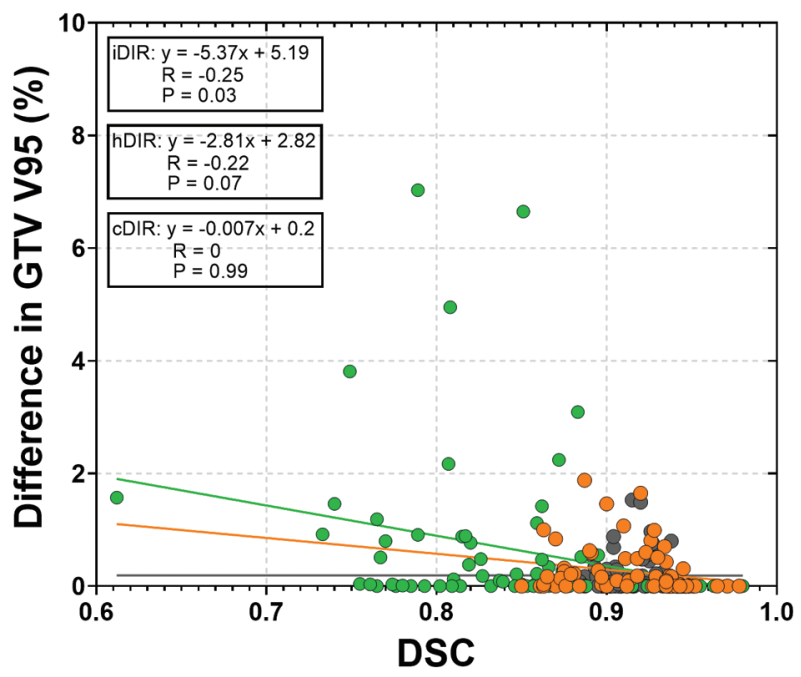

Figure 3. Dependence of dosimetric difference in clinical target volume (CTV) (A) and gross tumor volume (GTV) (B) on dice similarity coefficient (DSC).

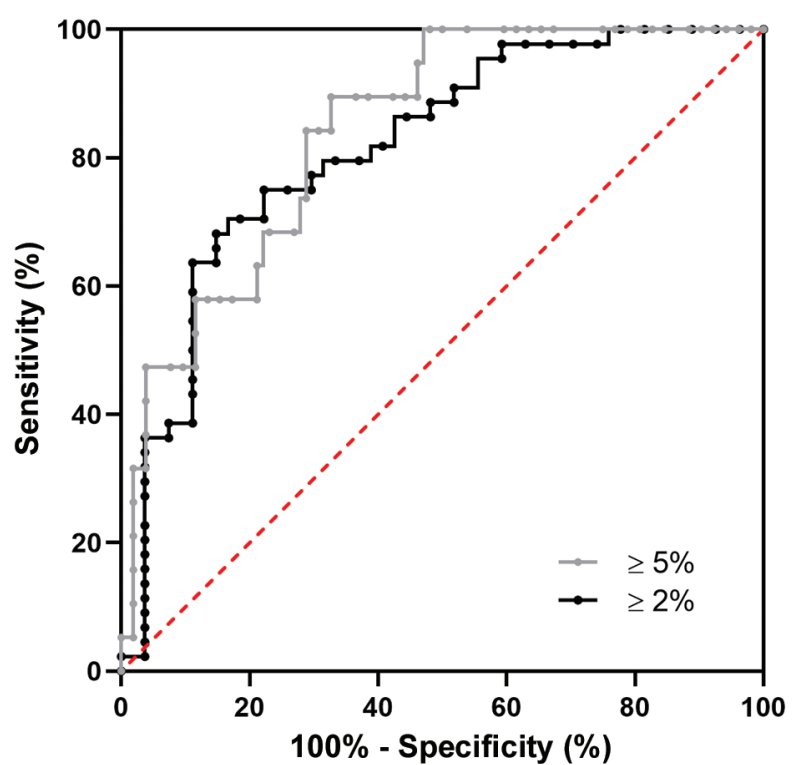

Figure 4. Receiver operating characteristic curves for dice similarity coefficients (DSCs) as predictors of dose differences for iDIR. The receiver operating characteristic curve for DSC with $2 \%$ (black line) and $5 \%$ (gray line) dose difference yielded an area under the curve of 0.817 and 0.843 , respectively. These were statistically significant $(p<0.0001)$.

Previous studies have reported that a poor DSC may be obtained using iDIR in the abdominal and pelvic region because of the large volume changes in the organs $(5,6,11$, 18). Indeed, the DSC is only about 0.7 for the rectum and bladder in prostate cancer $(11,18)$. In this study, using iDIR, a median DSC value of 0.89 and 0.82 was obtained for the

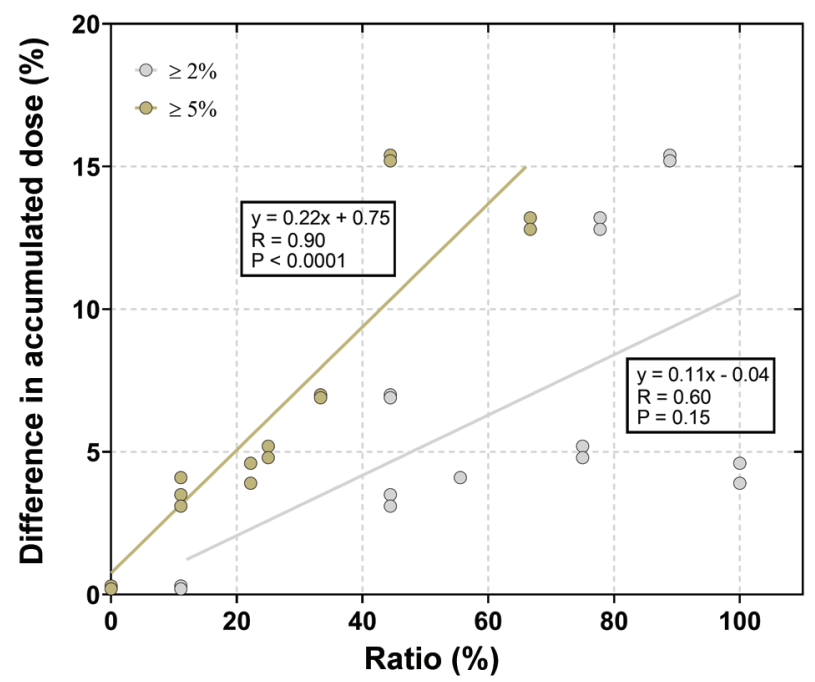

Figure 5. Dependence of the difference in accumulated dose in respect to the hDIR and cDIR on the percentage of cases with dosimetric changes greater than $2 \%$ and $5 \%$, respectively.

CTV and GTV, respectively, which were higher than the abovementioned studies. This is mainly because the CTV and GTV changes are relatively small in pancreatic cancer (Table I). Furthermore, satisfactory DSCs were obtained using hDIR and cDIR, especially for the CTV, in which the median values were 0.94 and 0.96 , respectively (Table II). In the current study, possible causes for a lower DSC for iDIR may be because the CTV margin was not defined based on an 
anatomical boundary, meaning that there was no clear intensity-based boundary. In addition, unlike the almost isotropic CTV boundary available in lung and liver cancer, the CTV margin of pancreatic cancer may vary with the anatomical changes surrounding the tumor. However, iDIR has limited capacity to estimate these changes in a low-contrast area (6), which may result in a poor deformed contour in some cases. hDIR and cDIR deform each point based on structures of the target and have no such limitation. For the GTV, the DSCs decreased in all DIR methods tested (Table III), suggesting that DIR accuracy is subject to the target volume. In general, DIR had a better performance in organs with larger volumes in the contour propagation process (19). Many improved algorithms and tools have been developed to reduce these inherent uncertainties and improve the accuracy of contour propagation for adaptive RT (20-22). In addition, the errors from inter- and intra-observer variation in target delineation should be considered (23). In this study, the contours on each CT set were examined by two radiation oncologists to reduce errors, and the average change in CTV was only $3.9 \mathrm{ml}$, and $2.4 \mathrm{ml}$ in GTV (Table I). However, case 4 has a relatively large change in CTV ( $25 \mathrm{ml}$ on average), which may have influenced the DIR accuracy.

We also investigated the discrepancy of warped dose among the DIR methods for CTV and GTV. Corroborating a prior study, a minimal dosimetry difference was observed in the CTV using cDIR and hDIR (5); this is mainly attributed to the satisfactory volume overlap of targets. However, a significant difference was observed between hDIR and CDIR $(p<0.05)$ (Table II). Therefore, cDIR appears to be the best DIR method for image registration in pancreatic cancer. In contrast, approximately $62 \%$ of cases had a difference greater than $2 \%$ in CTV V95 using iDIR (Figure 1). In other words, CT values should not be used for DIR of the CTV in pancreatic cancer, as they can cause a decrease in accuracy. Although we obtained a moderate negative dependence between the DSC and dose variation, it is important to note that a poor DSC does not always indicate a great change in dose parameter when the deformed volume is within the beam field. Thus, this dose deformation cannot represent the actual dose distribution and may cause overestimation of the dose to the surrounding organs. Therefore, we proposed a cut-off value to predict the dosimetry difference for iDIR, in which the dose changes would be greater than $2 \%(5 \%)$ in CTV V95 if the DSC was smaller than $0.91(0.89)$. This finding might be helpful for physicians to estimate the dose prior to the warping dose, as well as to help them select an adequate DIR method. For example, for case 5, cDIR and hDIR are recommended because the ratio over $2 \%(5 \%)$ of dose difference was $77.8 \%(66.7 \%)$ when using iDIR; however, for case 8 , iDIR seems a better choice since it has a similar DSC as the other DIR methods (the ratio over $2 \%$ and $5 \%$ of the dose difference was only $11.1 \%$ and 0 with
iDIR, respectively), but the registration process is much simpler. However, DSC can only be obtained after DIR, and other parameters that can provide guidance before applying DIR seem to be more effective, which will be examined in the future. For the GTV, decreased DSCs seem to have limited effects on the dose variations compared to the CTV because of the relatively large dose coverage. However, considering the steeper lateral dose penumbra applied in CIRT, large variations may be observed when the GTV margin is close to the CTV margin, so it merits close attention. In addition, to quantify the dosimetric difference based on the entire course of treatment, we calculated the accumulated dose using three types of DIR methods. From our results, using iDIR, the accumulated dose in the CTV V95 was underestimated by approximately $5 \%$ compared to hDIR and cDIR. Reducing the percentage of cases with a dose difference greater than 5\% appears to be crucial for the accumulated dose; therefore, ensuring a DSC of more than 0.89 is important. The results above can be used as a reference for dose accumulation for pancreatic cancer. However, considering that the impact of DIR methods on dose warping is patient and organ-specific, and the fact that only a few cases were analyzed in this study, a validation study is necessary in the future.

The present study has several limitations. First, we evaluated the DIR accuracy for the CTV and GTV only, and organs at risk, such as the duodenum and stomach, were not considered, which are also areas of concern in adaptive radiotherapy. However, DIR has limited capacity to achieve satisfactory accuracy for dose warping for organs with a high degree of deformation, especially for those that have a sharp dose gradient inside $(12,24)$; while biomechanical modelbased DIR may improve the accuracy, but currently only for bladder, rectum and parotid $(24,25)$. Second, we used DSC to evaluate the accuracy of volume registration, which has limitations in ensuring spatial accuracy. Unfortunately, it appears difficult to define anatomical landmarks for CTV and GTV in pancreatic cancer to validate the anatomical accuracy. Another limitation is that our results are based on single software. There may be variations in accuracy when employing different software $(7,26)$. However, similar results would be obtained when using a method based on the pixel values to evaluate the dose for pancreatic cancer.

Considering the inherent uncertainty of DIR, DIR algorithms should be selected carefully. In this study, we evaluated and quantified the DIR accuracy for three DIR methods based on adaptive CIRT. Compared to iDIR, both hDIR and cDIR have a superior accuracy in target structure and dose warping, especially in CTV. The proposed cut-off value may provide a reference for the selection of DIR method. iDIR may present a similar performance when the DSCs are greater than 0.89 . For the accumulated dose, all DIR methods presented a good performance for GTV; 
however, it may be underestimated by $5 \%$ for the CTV V95 using iDIR. Therefore, hDIR and cDIR (especially cDIR), are recommended for assessing the accumulated dose for pancreatic cancer with CIRT. Further study with a large sample and more evaluation metrics is necessary.

\section{Conflicts of Interest}

The Authors declare that the research was conducted in the absence of any commercial or financial relationships that could be construed as a potential conflict of interest.

\section{Authors' Contributions}

Conceptualization, YK.; methodology, YK, YL, and SO; software, YK, YL.; validation, YK. and YL.; formal analysis, YL.; investigation, YK, YL, MO, SS, SO, TM, and SK; resources, YK, YL; data curation, YK and YL; writing-original draft preparation, YL; writing-review and editing, YL, YK, SS, SO, TM, and MO; visualization, YK; supervision, $\mathrm{YK}, \mathrm{MO}$, and $\mathrm{TO}$; project administration, YK, MO, and TO.

\section{Acknowledgements}

The Authors thank the radiology technologists, medical doctors, nurses, and medical physicists at GHMC for their valuable insights.

\section{References}

1 Ohno T: Particle radiotherapy with carbon ion beams. EPMA J 4(1): 9, 2013. PMID: 23497542. DOI: 10.1186/1878-5085-4-9

$2 \mathrm{Li} \mathrm{Y,} \mathrm{Kubota} \mathrm{Y,} \mathrm{Tashiro} \mathrm{M} \mathrm{and} \mathrm{Ohno} \mathrm{T:} \mathrm{Value} \mathrm{of} \mathrm{three-}$ dimensional imaging systems for image-guided carbon ion radiotherapy. Cancers (Basel) 11(3): 297, 2019. PMID: 30832346. DOI: $10.3390 /$ cancers 11030297

3 Oh S and Kim S: Deformable image registration in radiation therapy. Radiat Oncol J 35(2): 101-111, 2017. PMID: 28712282. DOI: $10.3857 /$ roj.2017.00325

4 Liu F, Erickson B, Peng C and Li XA: Characterization and management of interfractional anatomic changes for pancreatic cancer radiotherapy. Int J Radiat Oncol Biol Phys 83(3): e423e429, 2012. PMID: 22436785. DOI: 10.1016/j.jirobp.2011.12.073

5 Kubota Y, Okamoto M, Li Y, Shiba S, Okazaki S, Komatsu S, Sakai M, Kubo N, Ohno T and Nakano T: Evaluation of intensity- and contour-based deformable image registration accuracy in pancreatic cancer patients. Cancers (Basel) 11(10): 1447, 2019. PMID: 31569617. DOI: 10.3390/cancers 11101447

6 Brock KK: Results of a multi-institution deformable registration accuracy study (MIDRAS). Int J Radiat Oncol Biol Phys 76(2): 583-596, 2010. PMID: 19910137. DOI: 10.1016/j.ijrobp.2009. 06.031

7 Pukala J, Johnson PB, Shah AP, Langen KM, Bova FJ, Staton RJ, Mañon RR, Kelly P and Meeks SL: Benchmarking of five commercial deformable image registration algorithms for head and neck patients. J Appl Clin Med Phys 17(3): 25-40, 2016. PMID: 27167256. DOI: 10.1120/jacmp.v17i3.5735

8 Nie K, Pouliot J, Smith E and Chuang C: Performance variations among clinically available deformable image registration tools in adaptive radiotherapy - How should we evaluate and interpret the result? J Appl Clin Med Phys 17(2): 328-340, 2016. PMID: 27074457. DOI: 10.1120/jacmp.v17i2.5778

9 Li Y, Kubota Y, Kubo N, Mizukami T, Sakai M, Kawamura, H, Irie D, Okano N, Tsuda K, Matsumura A, Saitoh JI, Nakano T and Ohno T: Dose assessment for patients with stage I non-small cell lung cancer receiving passive scattering carbon-ion radiotherapy using daily computed tomographic images: A prospective study. Radiother Oncol 144: 224-230, 2020. PMID: 32044421. DOI: 10.1016/j.radonc.2020.01.003

10 Kubota Y, Katoh H, Shibuya K, Shiba S, Abe S, Sakai M, Yuasa D, Tsuda K, Ohno T and Nakano T: Comparison between bone matching and marker matching for evaluation of intra- and interfractional changes in accumulated of carbon ion radiotherapy for hepatocellular carcinoma. Radiother Oncol 137: 77-82, 2019. PMID: 31078014. DOI: 10.1016/j.radonc.2019.04.026

11 Takayama Y, Kadoya N, Yamamoto T, Ito K, Chiba M, Fujiwara K, Miyasaka Y, Dobashi S, Sato K, Takeda K and Jingu K: Evaluation of the performance of deformable image registration between planning CT and CBCT images for the pelvic region: comparison between hybrid and intensity-based DIR. J Radiat Res 58(4): 567-571, 2017. PMID: 28158642. DOI: 10.1093/ jrr/rrw123

12 Jamema SV, Mahantshetty U, Andersen E, Noe K, Sørensen TS, Kallehauge JF, Shrivastava SK, Deshpande DD and Tanderup K: Uncertainties of deformable image registration for dose accumulation of high-dose regions in bladder and rectum in locally advanced cervical cancer. Brachytherapy 14(6): 953-962, 2015. PMID: 26489919. DOI: 10.1016/j.brachy.2015.08.011

13 Wognum S, Heethuis SE, Rosario T, Hoogeman MS and Bel A: Validation of deformable image registration algorithms on CT images of ex vivo porcine bladders with fiducial markers. Med Phys 41(7): 071916, 2014. PMID: 24989394. DOI: 10.1118/ 1.4883839

14 Jin J, McKenzie E, Fan Z, Tuli R, Deng Z, Pang J, Fraass B, Li D, Sandler H, Yang G, Sheng K, Gou S and Yang W: Nonlocal means denoising of self-gated and $\mathrm{k}$-space sorted 4-dimensional magnetic resonance imaging using block-matching and 3dimensional filtering: implications for pancreatic tumor registration and segmentation. Int J Radiat Oncol Biol Phys 95(3): 1058-1066, 2016. PMID: 27302516. DOI: 10.1016/ j.ijrobp.2016.02.006

15 Ziegler M, Nakamura M, Hirashima H, Ashida R, Yoshimura M, Bert $\mathrm{C}$ and Mizowaki T: Accumulation of the delivered treatment dose in volumetric modulated arc therapy with breathhold for pancreatic cancer patients based on daily cone beam computed tomography images with limited field-of-view. Med Phys 46(7): 2969-2977, 2019. PMID: 31055859. DOI: 10.1002/ mp.13566

16 Kanai T, Endo M, Minohara S, Miyahara N, Koyama-ito H, Tomura H, Matsufuji N, Futami Y, Fukumura A, Hiraoka T, Furusawa Y, Ando K, Suzuki M, Soga F and Kawachi K: Biophysical characteristics of HIMAC clinical irradiation system for heavy-ion radiation therapy. Int J Radiat Oncol Biol Phys 44(1): 201-210, 1999. PMID: 10219815. DOI: 10.1016/s03603016(98)00544-6

17 Kadoya N, Nakajima Y, Saito M, Miyabe Y, Kurooka M, Kito S, Fujita Y, Sasaki M, Arai K, Tani K, Yagi M, Wakita A, Tohyama $\mathrm{N}$ and Jingu K: Multi-institutional validation study of commercially available deformable image registration software 
for thoracic images. Int J Radiat Oncol Biol Phys 96(2): 422431, 2016. PMID: 27475673. DOI: 10.1016/j.ijrobp.2016.05.012

18 Thor M, Petersen JBB, Bentzen L, Høyer M and Muren LP: Deformable image registration for contour propagation from $\mathrm{CT}$ to cone-beam CT scans in radiotherapy of prostate cancer. Acta Oncol 50(6): 918-925, 2011. PMID: 21767192. DOI: 10.3109/ 0284186X.2011.577806

19 Kumarasiri A, Siddiqui F, Liu C, Yechieli R, Shah M, Pradhan D, Zhong H, Chetty IJ and Kim J: Deformable image registration based automatic CT to CT contour propagation for head and neck adaptive radiotherapy in the routine clinical setting. Med Phys 41(12): 121712, 2014. PMID: 25471959. DOI: $10.1118 / 1.4901409$

20 Elmahdy MS, Jagt T, Zinkstok RT, Qiao Y, Shahzad R, Sokooti $\mathrm{H}$, Yousefi S, Incrocci L, Marijnen CAM, Hoogeman M and Staring M: Robust contour propagation using deep learning and image registration for online adaptive proton therapy of prostate cancer. Med Phys 46(8): 3329-3343, 2019. PMID: 31111962. DOI: $10.1002 / \mathrm{mp} .13620$

21 Gu X, Dong B, Wang J, Yordy J, Mell L, Jia X and Jiang SB: A contour-guided deformable image registration algorithm for adaptive radiotherapy. Phys Med Biol 58(6): 1889-1901, 2013. PMID: 23442596. DOI: 10.1088/0031-9155/58/6/1889

22 Beasley WJ, Mcwilliam A, Slevin NJ, Mackay RI and van Herk M: An automated workflow for patient-specific quality control of contour propagation. Phys Med Biol 61(24): 8577-8586, 2016. PMID: 27880733. DOI: 10.1088/1361-6560/61/24/8577
23 van Herk M: Errors and margins in radiotherapy. Semin Radiat Oncol 14(1): 52-64, 2004. PMID: 14752733. DOI: 10.1053/ j.semradonc.2003.10.003

24 Qin A, Liang J, Han X, O'Connell N and Yan D: Technical Note: The impact of deformable image registration methods on dose warping. Med Phys 45(3): 1287-1294, 2018. PMID: 29297939. DOI: $10.1002 / \mathrm{mp} .12741$

25 Qin A, Ionascu D, Liang J, Han X, O'Connell N and Yan D: The evaluation of a hybrid biomechanical deformable registration method on a multistage physical phantom with reproducible deformation. Radiat Oncol 13(1): 240, 2018. PMID: 30514348. DOI: $10.1186 / \mathrm{s} 13014-018-1192-x$

26 Delpon G, Escande A, Ruef T, Darréon J, Fontaine J, Noblet C, Supiot S, Lacornerie $\mathrm{T}$ and Pasquier D: Comparison of automated atlas-based segmentation software for postoperative prostate cancer radiotherapy. Front Oncol 6: 178, 2016. PMID: 27536556. DOI: 10.3389/fonc.2016.00178
Received December 11, 2020

Revised December 28, 2020

Accepted December 29, 2020 